Pacific Journal of Mathematics

ON THE REPRESENTATION OF OPERATORS BY 


\title{
ON THE REPRESENTATION OF OPERATORS BY CONVOLUTION INTEGRALS
}

\author{
J. D. WestoN
}

1. Introduction. Let $\mathfrak{X}$ be the complex vector space consisting of all complex-valued functions of a non-negative real variable. For each positive number $u$, let the shift operator $I_{u}$ be the mapping of $\mathfrak{X}$ into itself defined by the formula

$$
I_{u} x(t)=\left\{\begin{array}{cl}
0 & (0 \leqq t<u) \\
x(t-u) & (t \geqq u)
\end{array}\right.
$$

Evidently, $I_{u+v}=I_{u} I_{v}$, for any positive numbers $u$ and $v$.

A linear operator $A$ which maps a subspace $\mathfrak{D}$ of $\mathfrak{X}$ into itself will here be called a $V$-operator (after Volterra) if

(1.1) for each $x$ in $\mathfrak{D}$, the conjugate function $x^{*}$ belongs to $\mathfrak{D}$,

(1.2) both $\mathfrak{D}$ and $\mathfrak{X} \mid \mathfrak{D}$ are invariant under the shift operators,

(1.3) every shift operator commutes with $A$.

Many operators that occur in mathematical physics are of this type. If $\mathfrak{D}$ is any subspace of $\mathfrak{X}$ having the properties (1.1) and (1.2), the restriction to $\mathfrak{D}$ of each shift operator is an example of a $V$-operator. All 'perfect operators' (of which a definition may be found in [5] $]^{1}$ are $V$-operators, on the space of perfect functions.

In this paper we obtain a representation theorem for $V$-operators which are continuous in a certain sense. This result leads to characterizations of two related classes of perfect operators, one of which has been considered from a different point of view in [5]. The main representation theorem (Theorem 4) is similar to a result obtained by R. E. Edwards [2] for $V$-operators which are continuous in another sense; and it closely resembles a theorem given recently by König and Meixner ([3], Satz 3).

2. Elementary properties of $\mathrm{V}$-operators. An important property of $V$-operators is given by

THEOREM 1. Let $A$ be a $V$-operator, and let $x_{1}$ and $x_{2}$ be two of its operands such that, for some positive number $t_{0}, x_{1}(t)=x_{2}(t)$ whenever $0 \leqq t \leqq t_{0}$. Then $A x_{1}(t)=A x_{2}(t)$ whenever $0 \leqq t \leqq t_{0}$.

Proof. Let $x=x_{1}-x_{2}$. Then, since $x(t)=0$ if $0 \leqq t \leqq t_{0}$, there is

1 And in $\$ 4$ below. 
a function $y$ such that $x=I_{t_{0}} y$; and $y$ is an operand of $A$, by virtue of the property (1.2). Consequently, by virtue of (1.3), $A x=I_{t_{0}} A y$; so that $A x(t)=0$ whenever $0 \leqq t \leqq t_{0}$. But $A x=A x_{1}-A x_{2}$, since $A$ is linear: hence the conclusion of the theorem.

With products and linear combinations defined in the usual way, the $V$-operators on a given space $\mathfrak{D}$ constitute a linear algebra $\mathfrak{A}(\mathfrak{D})$. If $A$ belongs to $\mathfrak{A}(\mathfrak{D})$ then so does the operator $A^{*}$ defined by

$$
A^{*} x=\left(A x^{*}\right)^{*},
$$

where $x$ is any function in $\mathfrak{D}$. We therefore have the unique decomposition

$$
A=B+i C,
$$

where $B$ and $C$ belong to $\mathfrak{A}(\mathfrak{D})$ and are 'real' in the sense that $B x$ and $C x$ are real for every real function $x$ in $\mathfrak{D}$. (The property (1.1) ensures that every function $x$ in $\mathfrak{D}$ can be uniquely expressed as $x_{1}+i x_{2}$, where $x_{1}$ and $x_{2}$ are real functions in $\mathfrak{D}$.)

If $A$ is a linear combination of shift operators, we have

$$
A=\sum_{j=1}^{n} \alpha_{j} I_{u_{j}}=I_{u} \sum_{j=1}^{n} \alpha_{j} I_{u_{j}-u},
$$

where $\alpha_{1}, \cdots, \alpha_{n}$ are complex numbers, $u$ is the least of the positive numbers $u_{1}, \cdots, u_{n}$, and $I_{0}$ is the unit operator (to be denoted henceforth by ' $I$ '). From this it is apparent that $A$ has no reciprocal in the algebra $\mathfrak{A}(\mathfrak{X})$; however, $I-A$ has a reciprocal in $\mathfrak{A}(\mathfrak{X})$, as the following result shows.

ThEOREM 2. Let $A$ be a $V$-operator on a space $\mathfrak{D}$, and let $u$ be any positive number. Then the formula

$$
B x(t)=x(t)+\sum_{n=1}^{\infty} I_{n u} A^{n} x(t),
$$

where $x$ is any function in $\mathfrak{D}$, and $t \geqq 0$, defines a linear transformation $B$, of $\mathfrak{D}$ into $\mathfrak{X}$, which commutes with every shift operator and is such that $B\left(I-I_{u} A\right) x=x$ for every $x$ in $\mathfrak{D}$ and $\left(I-I_{u} A\right) B x=x$ if $B x$ is in D.

Proof. The series defining $B$ certainly converges (pointwise): in fact, if $t_{0} \geqq 0$ and $m$ is a positive integer such that $m u \geqq t_{0}$, then, for any $x$ in $\mathfrak{D}$,

$$
B x(t)=x(t)+\sum_{n=1}^{m} I_{n u} A^{n} x(t)
$$


whenever $0 \leqq t \leqq t_{0}$. Hence if $B x$ is in $\mathfrak{D}$ then, by Theorem 1 ,

$$
\left(I-I_{u} A\right) B x(t)=x(t)-I_{(m+1) u} A^{m+1} x(t)=x(t)
$$

whenever $0 \leqq t \leqq t_{0}$; so that $\left(I-I_{u} A\right) B x=x$, since $t_{0}$ is arbitrary. Also, if $x$ is in $\mathfrak{D}$ then $\left(I-I_{u} A\right) x$ is in $\mathfrak{D}$, so that

$$
\begin{aligned}
B\left(I-I_{u} A\right) x(t) & =\left(I-I_{u} A\right) x(t)+\sum_{n=1}^{m} I_{n u} A^{n}\left(I-I_{u} A\right) x(t) \\
& =x(t)-I_{(m+1) u} A^{m+1} x(t)=x(t)
\end{aligned}
$$

whenever $0 \leqq t \leqq t_{0}$. Thus $B\left(I-I_{u} A\right) x=x$. It can be verified in a similar way that $B$ commutes with the shift operators and is linear.

If the transformation $B$ of Theorem 2 maps $D$ into itself, then $I-I_{u} A$ has a reciprocal in $\mathfrak{A}(\mathfrak{D})$, namely $B$. This is certainly the case if $\mathfrak{D}$ consists of all the functions $x$ that have some purely local property (for example, continuity, with $x(0)=0$, or differentiability, with $x(0)=x^{\prime}(0)=0$, or local integrability). ${ }^{2}$ It is also the case with certain other choices of D), provided that $A$ is restricted to be a linear combination of shift operators; for example, if $(\mathcal{D}$ consists of the perfect functions, then an operator of the form

$$
\alpha_{0} I+\alpha_{1} I_{u_{1}}+\cdots+\alpha_{n} I_{u_{n}}
$$

has a reciprocal in $\mathfrak{A}(\mathfrak{D})$ if $\alpha_{0} \neq 0$ (this can be seen at once on taking Laplace transforms and using Theorem 6 of [5]).

If $(\mathcal{D}$ contains more than the zero function, it is clear that (2.1) represents the zero operator on $\mathfrak{D}$ only if all the coefficients $\alpha_{0}, \cdots, \alpha_{n}$ are zero; and since the product of two operators of this form is another such operator, the reciprocal of (2.1) cannot be expressed in the same form unless it is a scalar multiple of $I$. Thus it is usual for $\mathfrak{A}(\mathfrak{D})$ to contain operators other than those of the form (2.1). In general it seems to be difficult to decide whether $\mathfrak{A}(\mathfrak{D})$ is commutative or not; but it is shown in $\S 4$ that $\mathfrak{D}$ can be chosen, of moderate size, so that $\mathfrak{A}(\mathfrak{D})$ is not commutative.

The Laplace transformation is naturally associated with the idea of a $V$-operator, because it converts the shift operators to exponential factors. A locally integrable function $x$ has an absolutely convergent Laplace integral if $x$ is of exponential order at infinity, in the sense that $x(t)=O\left(e^{c t}\right)$ as $t \rightarrow \infty$, for some real number $c$ (depending on $x$ ). One can consider $V$-operators on spaces consisting of such functions, and for some of these spaces the following result is available.

THeOREM 3. Let $A$ be a $V$-operator on a space D consisting of all

${ }^{2}$ A property at infinity might be regarded as 'local', but this interpretation is to be excluded here. 
the functions in $\mathfrak{X}$ which satisfy some (possibly empty) set of local conditions and are of exponential order at infinity. Then there are positive numbers $b$, $c$, and $\tau$ such that $|A x(t)| \leqq b e^{c t}$ whenever $t \geqq \tau$ and $|x(t)| \leqq 1$ for all $t$, with $x$ in $\mathfrak{D}$.

Proof. Assuming the theorem to be false, we shall construct inductively a sequence $\left\{x_{n}\right\}$ in $\mathfrak{D}$, and a sequence $\left\{t_{n}\right\}$ of positive numbers, such that, for each positive integer $n$,

(i) $\left|x_{n}(t)\right| \leqq 2^{-n}$ for all values of $t$,

(ii) $t_{n} \geqq n$,

(iii) $x_{n}(t)=0$ if $0 \leqq t \leqq t_{n-1}$, where $t_{0}=0$,

(iv) $\left|\sum_{j=1}^{n} A x_{j}\left(t_{n}\right)\right| \geqq e^{n t_{n}}$.

In the first place, if the theorem is false, we can choose $x_{1}$ so that $\left|x_{1}(t)\right| \leqq \frac{1}{2}$ for all values of $t$ and $\left|A x_{1}(t)\right| \geqq e^{t}$ for some value of $t$, say $t_{1}$, greater than 1. Suppose, then, that the first $m-1$ terms of each sequence have been chosen, where $m>1$, so that (i)-(iv) hold when $n \leqq m-1$. Let

$$
y_{m}=\sum_{j=1}^{m-1} A x_{j} .
$$

Since $y_{m}$ belongs to $\mathfrak{D}$, there is a real number $c_{m}$ such that $\left|y_{m}(t)\right| \leqq$ $e^{c_{m}{ }^{t}}$ when $t$ is sufficiently large. We can choose $x_{m}$ so that $\left|x_{m}(t)\right| \leqq 2^{-m}$ for all $t, x_{m}(t)=0$ if $0 \leqq t \leqq t_{m-1}$, and

$$
\left|A x_{m}\left(t_{m}\right)\right| \geqq 2 e^{\left(c_{m}+m\right) t_{m}},
$$

where $t_{m}$ is chosen so that $t_{m} \geqq m$ and $\left|y_{m}\left(t_{m}\right)\right| \leqq e^{c_{m} t_{m}}$. Then

$$
\left|\sum_{j=1}^{m} A x_{j}\left(t_{m}\right)\right| \geqq\left|A x_{m}\left(t_{m}\right)\right|-\left|y_{m}\left(t_{m}\right)\right| \geqq e^{\left(c_{m}+m\right) t_{m}} \geqq e^{m t_{m}}
$$

Thus (i)-(iv) hold when $n=m$.

Now let $x_{0}=\sum_{n=1}^{\infty} x_{n}$. Then $\left|x_{0}(t)\right| \leqq 1$ for all $t$, by virtue of (i); and $x_{0}$ belongs to $\mathfrak{D}$ since, by (iii), it has the appropriate local properties. Hence there is a real number $c_{0}$ such that $A x(t)=O\left(e^{c_{0} t}\right)$ as $t \rightarrow \infty$; so that, by (ii), $A x\left(t_{n}\right)=O\left(e^{c_{0} t_{n}}\right)$ as $n \rightarrow \infty$. But, by (iii) and (iv), and Theorem $1,\left|A x\left(t_{n}\right)\right| \geqq e^{n t_{n}}$ for each $n$. This contradiction proves the theorem.

3. Strong continuity. If the field of complex numbers is given either the discrete topology or the usual topology, the space $\mathfrak{X}$ can be given the corresponding topology of uniform convergence on finite closed intervals. The first of these topologies for $\mathfrak{X}$ has the property that every $V$-operator is continuous with respect to it, as Theorem 1 shows; but it does not make $\mathfrak{X}$ a topological vector space (it has the defect that $n^{-1} x \rightarrow 0$ as $n \rightarrow \infty$ only if $x$ is the zero function). The second topology for $\mathfrak{X}$ 
is more interesting, and will be referred to as the strong topology. In fact we shall consider this only in relation to the closed subspace, $\mathfrak{b}_{0}$, consisting of all the continuous functions $x$ for which $x(0)=0$. For each $x$ in $\mathfrak{F}_{0}$, and each non-negative number $t$, we define $\|x\|_{t}$ to be the least upper bound of $|x(u)|$ with $0 \leqq u \leqq t$. We can then give $\widetilde{\Xi}_{0}$ a metric, which determines the strong topology, by taking the distance between functions $x$ and $y$ to be

$$
\sum_{n=1}^{\infty} 2^{-n}\|x-y\|_{n} /\left(1+\|x-y\|_{n}\right) .
$$

In this way $\mathfrak{c}_{0}$ becomes a Fréchet space.

In the case of $\mathfrak{C}_{0}$, which is an example of a space $\mathfrak{D}$ satisfying (1.1) and (1.2), a large class of $V$-operators, including those of the form (2.1), can be defined in terms of Riemann-Stieltjes convolution integrals. If $\nu$ is a function which belongs to $\mathfrak{X}$ and has bounded variation in every finite interval $[0, t]$, then the formula

$$
A x(t)=\int_{0}^{t} x(t-u) d \nu(u)
$$

where $x$ is any function in $\mathfrak{S}_{0}$, defines a $V$-operator $A$ on $\mathfrak{\Im}_{0}$ (cf. [5], Theorem 3). Moreover, if $0 \leqq v \leqq t$ then

$$
|A x(v)| \leqq \int_{0}^{v}\left|x ( v - u ) \left\|d \nu(u)\left|\leqq \int_{0}^{t}\|x\|_{t}\right| d \nu(u) \mid, \quad(t \geqq 0),\right.\right.
$$

so that

$$
\|A x\|_{t} \leqq\|x\|_{t} \int_{0}^{t}|d \nu(u)|
$$

whence it follows that $A$ is strongly continuous (continuous with respect to the strong topology). The theorem we are about to prove shows that every strongly continuous $V$-operator on a sufficiently large space $D$ of continuous functions can be represented in this way (and can therefore be extended from $D$ to the whole of $\left(\mathbb{S}_{0}\right)$.

If $A$ is a linear operator on a subspace $\mathfrak{D}$ of $\mathfrak{F}_{0}$, and if $t \geqq 0$, we denote by ' $\|A\|_{t}$ ' the least upper bound of $\|A x\|_{t}$ with $x$ in $\mathfrak{D}$ and $\|x\|_{t} \leqq 1$. It is clear that $A$ is strongly continuous if and only if $\|A\|_{t}$ is finite for all values of $t$ (or, equivalently, for all sufficiently large values of $t$ ).

THEOREM 4. Let $A$ be a strongly continuous $V$-operator on a strongly dense subspace $\mathfrak{D}$ of $\mathfrak{S}_{0}$, and let $t$ be any positive number. Then there is a function $\nu$ in $\mathfrak{X}$, with $\nu(0)=0$ and $\nu(u-)=\nu(u)$ whenever $0<u \leqq t$, such that $A x(t)$ is given by (3.1) for every $x$ in $\mathfrak{D}$. This function $\nu$ is uniquely determined by $A$, and is independent of $t$; its total variation 
in the interval $[0, t]$ is $\|A\|_{t}$.

Proof. For each function $x$ in $\mathfrak{D}$, and for each positive number $t$, let $x_{t}$ be the restriction of $x$ to the closed interval $[0, t]$. Then, for a fixed value of $t$, the mapping $x \rightarrow x_{t}$ is a linear transformation of $\mathfrak{D}$ on to a subspace $\mathfrak{D}_{t}$ of the complex Banach space $C[0, t]$, consisting of all continuous functions on the interval $[0, t]$; moreover, $\left\|x_{t}\right\|=\|x\|_{t}$. If $x_{t}=0$ then $A x(t)=0$, by Theorem 1; we can therefore define a linear functional $\phi$ on $\mathfrak{D}_{t}$ by the formula

$$
\varphi\left(x_{t}\right)=A x(t) .
$$

This functional is continuous, with $\|\varphi\|=\|A\|_{t}$.

An integral representation of $\varphi$ can be found by adapting a construction used by Banach ([1], 59-60). By a well-known theorem ${ }^{3}, \varphi$ can be extended without change of norm to the complex Banach space $M[0, t]$, which contains the characteristic functions of all the subintervals of $[0, t]$. A function $\nu_{t}$ can then be defined on $[0, t]$ so that $\nu_{t}(0)=0$ and

$$
\begin{gathered}
\int_{0}^{t}\left|d \nu_{t}(u)\right| \leqq\|\varphi\|, \\
\varphi(f)=\int_{0}^{t} f(t-u) d \nu_{t}(u)
\end{gathered}
$$

for every function $f$ in $C[0, t]$.

Without affecting the validity of (i) or (ii), we can adjust $\nu_{t}$ so that it is continuous on the left at each interior point of the interval $[0, t]$. Moreover, if $f$ is a continuous function such that $f(0)=0$, then the jump of $\nu_{t}$ at the point $t$ makes no contribution to the integral in (ii); therefore, as far as such functions $f$ are concerned, we may suppose $\nu_{t}$ chosen so that $\nu_{t}(t-)=\nu_{t}(t)$, giving left-hand continuity throughout the interval $(0, t]$, and retaining (i). Under these conditions, $\nu_{t}$ is uniquely determined by $A$. For, if $0<v \leqq t$ and $0<\delta<v$, there is a function $f_{\delta}$ in $C[0, t]$ such that $\left\|f_{\delta}\right\|=1$ and

$$
f_{\delta}(u)= \begin{cases}0 & (0 \leqq u \leqq t-v) \\ 1 & (t-v+\delta \leqq u \leqq t)\end{cases}
$$

Thus

$$
\varphi\left(f_{\delta}\right)=\int_{0}^{v-\delta} d \nu_{t}(u)+\int_{v-\delta}^{v} f_{\delta}(t-u) d \nu_{t}(u)
$$

and therefore

$$
\left|\varphi\left(f_{\delta}\right)-\nu_{t}(v-\delta)\right| \leqq \int_{v-\delta}^{v}\left|d \nu_{t}(u)\right|,
$$

3 The Hahn-Banach-Bohnenblust-Sobczyk extension theorem: see, for example, [8], 113. 
so that $\varphi\left(f_{\delta}\right) \rightarrow \nu_{t}(v)$ as $\delta \rightarrow 0 .{ }^{4} \quad$ But since $\mathscr{D}$ is strongly dense in $\mathfrak{S}_{0}, f_{\delta}$ belongs to the closure of $\mathfrak{D}_{t}$, in $C[0, t]$; so that, $\varphi$ being continuous, $\varphi\left(f_{\delta}\right)$ is uniquely determined by $A$, for each value of $\delta$. This establishes the uniqueness of $\nu_{t}$.

Now suppose that $t^{\prime}>t$. By what has been proved, we have, for any $x$ in $\mathfrak{D}$,

$$
A x(t)=\int_{0}^{t} x(t-u) d \nu_{t}(u) .
$$

But $A x(t)=I_{t^{\prime}-t} A x\left(t^{\prime}\right)$, and $I_{t^{\prime}-t} A=A I_{t^{\prime}-t}$; hence

$$
A x(t)=\int_{0}^{t^{\prime}} I_{t^{\prime}-t} x\left(t^{\prime}-u\right) d \nu_{t^{\prime}}(u)=\int_{0}^{t} x(t-u) d \nu_{t^{\prime}}(u) .
$$

It follows that $\nu_{t}(u)=\nu_{t^{\prime}}(u)$ whenever $0 \leqq u \leqq t$; in particular, $\nu_{t}(t)=$ $\nu_{t^{\prime}}(t)$. Hence if we define the function $\nu$ by

$$
\nu(t)=\nu_{t}(t)
$$

we obtain the required representation of $A$.

Finally, (i) shows that

$$
\int_{0}^{t}|d \nu(u)| \leqq\|A\|_{t},
$$

and we have previously noted that, for any $x$ in $\mathfrak{D}$,

$$
\|A x\|_{t} \leqq\|x\|_{t} \int_{0}^{t}|d \nu(u)|
$$

Thus $\int_{0}^{t}|d \nu(u)|=\|A\|_{t}$, and the proof is complete. ${ }^{5}$

As a corollary, we have

THEOREm 5. Suppose that the formula

$$
A x(t)=\int_{0}^{t} K(t, u) x(u) d u
$$

defines a V-operator $A$ on $\mathbb{E}_{0}$, the kernel $K$ being such that $\int_{0}^{t}|K(t, u)| d u$ exists as a Lebesgue integral which is locally bounded with respect to $t$. Then there is a function $k$ in $\mathfrak{X}$ such that, for each $t, K(t, u)=k(t-u)$ for almost all values of $u$.

${ }^{4}$ Here we use the fact that if a function of bounded variation is continuous on the left, then so is its total variation.

${ }^{5}$ In this proof we have not fully used the fact that $A$ maps $D$ into itself: it is enough that $A$ maps $\mathfrak{D}$ into $C_{0}$. 
Proof. For each $t$, let $\|K\|_{t}$ be the least upper bound of $\int_{0}^{v}|K(v, u)| d u$ with $0 \leqq v \leqq t$; this is finite, by hypothesis. Then, for each $x$ in $\mathfrak{E}_{0}$,

$$
\|A x\|_{t} \leqq\|K\|_{t}\|x\|_{t}
$$

so that $A$ is strongly continuous. But

$$
A x(t)=\int_{0}^{t} K(t, t-u) x(t-u) d u,
$$

so that if

$$
L_{t}(u)=\int_{0}^{u} K(t, t-v) d v
$$

then

$$
A x(t)=\int_{0}^{t} x(t-u) d L_{t}(u) .
$$

Hence, by Theorem $4, L_{t}=\nu$, a function which is independent of $t$. Since $\nu$ has bounded variation, there is a function $k$ such that

$$
k(u)=\frac{d}{d u} \nu(u)
$$

except when $u$ is in a set $E$ whose Lebesgue measure is 0 . However, for each value of $t$,

$$
\frac{d}{d u} \nu(u)=\frac{d}{d u} L_{t}(u)=K(t, t-u)
$$

except when $u$ is in a set $E_{t}$ of measure 0 . Thus

$$
K(t, u)=k(t-u)
$$

except when $u$ is in the set $t-\left(E_{t} \cup E\right)$, which has measure 0 .

The functions in $\mathfrak{S}_{0}$ which are of exponential order at infinity form a subspace $\mathfrak{F}_{0}$. The perfect functions form a smaller subspace, $\mathfrak{D}_{0}$ (in fact $\mathfrak{D}_{0}$ is the largest subspace of $\mathfrak{F}_{0}$ which is invariant under the differential operator, $D$ ).

THEOREM 6. $\mathfrak{D}_{0}$ is strongly dense in $\mathfrak{F}_{0}$.

Proof. It is easily seen that $\mathfrak{F}_{0}$ is strongly dense in $\mathfrak{F}_{0}$ : in fact, if $x$ is in $\mathfrak{S}_{0}$ and $x_{n}$ is defined by

$$
x_{n}(t)= \begin{cases}x(t) & (0 \leqq t \leqq n) \\ x(n) & (t \geqq n),\end{cases}
$$


then $x_{n}$ belongs to $\breve{F}_{0}$, for each $n$, and $x_{n} \rightarrow x$ strongly as $n \rightarrow \infty$. To show that $\mathfrak{D}_{0}$ is dense in $\mathfrak{F}_{0}$, let $x$ be any function in $\mathfrak{F}_{0}$ and, for each positive number $\delta$, let $g_{(\delta)}$ be a positive perfect function such that if $t \geqq \delta$ then $g_{(\delta)}(t)=0$ and $\int_{0}^{t} g_{(\delta)}(u) d u=1$ (for example, we could take $g_{(\delta)}$ to be $D h_{(\delta)}$, where $h_{(\delta)}$ is given by Lemma 1 of [5]). Let $x_{(\delta)}=x * g_{(\delta)}$. Then $x_{(\delta)}$ belongs to $\mathfrak{D}_{0}$ (' $x *$ ' is a perfect operator), and, if $v \geqq \delta$,

$$
\begin{aligned}
x_{(\delta)}(v)-x(v) & =\int_{0}^{v} x(v-u) g_{(\delta)}(u) d u-x(v) \\
& =\int_{0}^{\delta}\{x(v-u)-x(v)\} g_{(\delta)}(u) d u .
\end{aligned}
$$

Now let $t$ and $\varepsilon$ be any positive numbers. Since $x$ is uniformly continuous in the interval $[0, t]$, with $x(0)=0$, we can choose $\delta$ so that

$$
|x(v-u)-x(v)|<\varepsilon
$$

whenever $\delta \leqq v \leqq t$, and $|x(v)|<\frac{1}{2} \varepsilon$ whenever $0 \leqq v \leqq \delta$; then

$$
\left|x_{(\delta)}(v)-x(v)\right|<\varepsilon \int_{0}^{\delta} g_{(\delta)}(u) d u=\varepsilon
$$

if $\delta \leqq v \leqq t$, and if $0 \leqq v \leqq \delta$,

$$
\begin{gathered}
\left|x_{(\delta)}(v)-x(v)\right| \leqq \int_{0}^{\delta}|x(v-u)| g_{(\delta)}(u) d u+|x(v)| \\
\leqq \frac{1}{2} \varepsilon \int_{0}^{\delta} g_{(\delta)}(u) d u+\frac{1}{2} \varepsilon=\varepsilon .
\end{gathered}
$$

Thus $\left\|x_{(\delta)}-x\right\|_{t}<\varepsilon$. It follows that $\mathfrak{D}_{0}$ is strongly dense in $\mathfrak{S}_{0}$.

In [5] it is shown that any positive perfect operator has the representation (3.1), with $\nu$ a non-decreasing function (in fact this holds for any positive $V$-operator on a space $\mathfrak{D}$ such that $\mathfrak{D}_{0} \subseteq \mathfrak{D} \subseteq\left(\mathfrak{S}_{0}\right)$. It follows that the linear combinations of positive perfect operators, which form a linear algebra $\mathfrak{M}\left(\mathfrak{D}_{0}\right)^{6}$, are strongly continuous. On the other hand, there are strongly continuous perfect operators which do not belong to $\mathfrak{M}\left(\mathfrak{D}_{0}\right)$ : for example, if $\nu(t)=\sin \left(e^{t^{2}}-1\right)$, and $A$ is defined on $\mathfrak{D}_{0}$ according to (3.1), then, as is shown in [5], $A$ is a perfect operator which is not in $\mathfrak{M}\left(\mathfrak{D}_{0}\right)$; but of course $A$ is strongly continuous. However, it is possible to characterize $\mathfrak{M}\left(\mathfrak{D}_{0}\right)$ in terms of seminorms, as follows.

Theorem 7. A V-operator $A$ on $\mathfrak{D}_{0}$ is an element of $\mathfrak{M}\left(\mathfrak{D}_{0}\right)$ if and only if there is a real number $c$ such that $\|A\|_{t}=O\left(e^{c t}\right)$ as $t \rightarrow \infty$.

Proof. By Theorem 1 of [5], an operator $A$ on $\mathfrak{D}_{0}$ is in $\mathfrak{M}\left(\mathfrak{D}_{0}\right)$ if 
and only if it admits the representation (3.1) with $\nu$ a linear combination of positive non-decreasing functions which are of exponential order at infinity. This condition on $\nu$ is equivalent to the existence of a real number $c$ such that $\int_{0}^{t}|d \nu(u)|=O\left(e^{c t}\right)$ as $t \rightarrow \infty$. Therefore, by Theorems 4 and 6 above, $A$ is in $\mathfrak{M}\left(\mathfrak{D}_{0}\right)$ if and only if $\|A\|_{t}=O\left(e^{c t}\right)$ as $t \rightarrow \infty$.

Each function $y$ in $\mathfrak{S}_{0}$ determines a strongly continuous $V$-operator $A$ on $\mathbb{S}_{0}$ according to the formula $A x=x * y$; for, integration by parts shows that this formula is equivalent to (3.1), with

$$
\nu(t)=D^{-1} y(t)=\int_{0}^{t} y(u) d u \quad(t \geqq 0) .
$$

An important property of convolution in $\mathfrak{\Im}_{0}$ is the fact that it obeys the associative law (as well as the commutative law); more generally, we have

THEOREM 8. Let $A$ and $B$ be strongly continuous $V$-operators, on $\mathfrak{S}_{0}$ and on a subspace $\mathfrak{D}$ of $\mathfrak{S}_{0}$ respectively. If $x$ is any function in $(\mathfrak{D}$ then $A x$ belongs to the strong closure of $\mathfrak{D}$; if $A x$ is in $\mathfrak{D}$ itself, then $A B x=B A x$. In particular, if $y$ is a function in $\mathfrak{S}_{0}$ such that $x * y$ is in $\mathfrak{D}$, then $B(x * y)=(B x) * y$.

Proof. Let $A$ be represented by a function $\nu$ in accordance with Theorem 4. Then for any $x$ in $\mathfrak{D}$, each value $A x(t)$ can be arbitrarily approximated by sums of the form

$$
\sum_{j=1}^{n}\left\{\nu\left(u_{j}\right)-\nu\left(u_{j-1}\right)\right\} x\left(t-u_{j}\right),
$$

where $0 \leqq u_{1} \leqq \cdots \leqq u_{n} \leqq t$; and this approximation is locally uniform with respect to $t$. Now the above sum is the value at $t$ of the function

$$
\sum_{j=1}^{n} \alpha_{j} I_{u_{j}} x,
$$

where $\alpha_{j}=\nu\left(u_{j}\right)-\nu\left(u_{j-1}\right)$. This function belongs to $\mathfrak{D}$, since $\mathfrak{D}$ satifies (1.2). Thus $A x$ belongs to the strong closure of $\mathfrak{D}$. Further, the points $u_{j}$ can be chosen in such a way that, while $A x$ is strongly approximated by (i), $A B x$ is simultaneously approximated, in the same sense, by

$$
\sum_{j=1}^{n} \alpha_{j} I_{u_{j}} B x \text {. }
$$

But, since $B$ is a $V$-operator, (ii) is the same as

$$
B \sum_{j=1}^{n} \alpha_{j} I_{u_{j}} x
$$


Since $B$ is strongly continuous, it follows that $A B x=B A x$ if $A x$ is an operand of $B$.

We can now prove a partial converse of Theorem 1, namely.

THEOREM 9. Let $A$ be a non-zero strongly continuous $V$-operator on $\mathfrak{c}_{0}$. Then there is a non-negative number $\tau$ such that (i) for any function $x$ in $C_{0}, A x(t)=0$ whenever $0 \leqq t \leqq \tau$, and (ii) if $A x(t)=0$ whenever $0 \leqq t \leqq t_{0}$, where $x$ belongs to $\mathfrak{S}_{0}$ and $t_{0} \geqq \tau$, then $x(t)=0$ whenever $0 \leqq t \leqq t_{0}-\tau$. In particular, $x=0$ if $A x=0$.

Proof. Let $\nu$ be the function representing $A$ according to Theorem 4 , and let $\tau$ be the greatest lower bound of the numbers $t$ for which $\nu(t) \neq 0$. Obviously, $\tau$ has the property (i) required by the theorem. Suppose that $x$ is a function in $\mathfrak{S}_{0}$ such that $A x(t)=0$ whenever $0 \leqq t \leqq t_{0}$, where $t_{0} \geqq \tau$. Let $g_{(\delta)}$ be defined as in the proof of Theorem 6 , and let $x_{(\delta)}=x * g_{(\delta)}$. Then, for each value of $\delta, x_{(\delta)}$ has a derivative $x_{(\delta)}^{\prime}$ in $\varepsilon_{0}$; in fact $x_{(\delta)}^{\prime}=x * g_{(\delta)}^{\prime}$. Also, if $0 \leqq t \leqq t_{0}$,

$$
\begin{aligned}
\int_{0}^{t} x_{(\delta)}^{\prime}(t-u) \nu(u) d u & =A x_{(\delta)}(t)=(A x) * g_{(\delta)}(t) \\
& =\int_{0}^{t} A x(t-u) g_{(\delta)}(u) d u=0 .
\end{aligned}
$$

Therefore, by a theorem of Titchmarsh [4, 327], $x_{(\delta)}^{\prime}(t)=0$ whenever $0 \leqq t \leqq t_{0}-\tau$ (we cannot have $\nu(t)=0$ for almost all $t$ in a neighbourhood of $\tau$, since $\nu$ is continuous on the left). Hence $x_{(\delta)}(t)=0$ whenever $0 \leqq t \leqq t_{0}-\tau$. Since $x_{(\delta)}(t) \rightarrow x(t)$ as $\delta \rightarrow 0$, the theorem follows.

It is a consequence of Theorem 8 that every strongly continuous $V$-operator on $\mathfrak{D}_{0}$ is a perfect operator (the converse is false; in fact it is easy to see that the differential operator $D$ is not strongly continuous). Thus an operator $A$ represented by (3.1) is a perfect operator if and only if it maps $\mathfrak{D}_{0}$ into itself. An equivalent condition is given by

THEOREM 10. The formula (3.1), with $x$ in $\mathfrak{D}_{0}$, represents a perfect operator $A$ if and only if there is a positive integer $n$ such that $D^{-n} \nu$ belongs to $\mathfrak{F}_{0}$, where

$$
D^{-n} \nu(t)=\int_{0}^{t} \cdots \int_{0}^{u_{2}} \nu\left(u_{1}\right) d u_{1} \cdots d u_{n} \quad\left(t=u_{n+1} \geqq 0\right) .
$$

Proof. For any perfect function $x$ and any positive integer $n$, we have from (3.1), after integration by parts, 


$$
A x(t)=\int_{0}^{t} x^{(n+1)}(t-u) D^{-n} \mathcal{\nu}(u) d u \quad(t \geqq 0) .
$$

Thus if $D^{-n} \nu$ belongs to $\xi_{0}$ for some value of $n$, then $A$ is a perfect operator. On the other hand, suppose that $A$, given by (3.1), is a perfect operator (when restricted to $\mathfrak{D}_{0}$ ). By a general representation theorem for perfect operators [6], there is a function $y$ in $\xi_{0}$ such that, for some positive integer $n$, and every perfect function $x$,

$$
A x(t)=\int_{0}^{t} x^{(n+1)}(t-u) y(u) d u \quad(t \geqq 0) .
$$

Hence $x^{(n+1)} *\left(y-D^{-n} \nu\right)=0$, so that, by Theorem $9, y=D^{-n} \nu$.

If $\nu(t)=e^{e^{t}}$, the $V$-operator $A$ given by (3.1) does not map $\mathfrak{D}_{0}$ into itself, since $\nu$ does not satisfy the condition of Theorem 10 .

Every perfect operator $A$ has a Laplace transform, $\bar{A}$ : if $A$ is given by (3.1), $\bar{A}$ may or may not be given by

$$
\bar{A}(z)=\int_{0}^{\infty} e^{-z t} d \nu(t),
$$

the integral being convergent when $\Re z$ is sufficiently large. This representation of $\bar{A}$ is certainly valid if $A$ belongs to $\mathfrak{M}\left(\mathfrak{D}_{0}\right)$ (cf. [5], Theorem 4); and also if $\nu(t)=\sin \left(e^{t^{2}}-1\right)$, for exa mple. But if $D^{-1} \nu(t)=\sin \left(e^{t^{2}}-1\right)$ the integral in (3.2) does not converge for any value of $z$ (as can be seen on integrating twice by parts). However, (3.2) holds whenever the integral is convergent, as the following result shows.

THEOREM 11. Let $A$ be any strongly continuous perfect operator, and let $\nu$ be a function such that $A$ is represented by (3.1). Then the Laplace transform $\bar{A}$ is represented by (3.2), with $\Re z$ sufficiently large, if the infinite integral is interpreted in the sense of summability $(C, n)$, where $n$ is any non-negative integer such that $D^{-n} \nu$ belongs to $\xi_{0}$.

Proof. Let $B$ be the perfect operator obtained on replacing $\nu$ by $D^{-1} \nu$ in (3.1). Then, if $x$ is any perfect function, and $t \geqq 0$,

$$
D B x(t)=B x^{\prime}(t)=\int_{0}^{t} x^{\prime}(t-u) \nu(u) d u=\nu(0) x(t)+\int_{0}^{t} x(t-u) d \nu(u) .
$$

Thus $D B=\nu(0) I+A$. If $\nu$ belongs to $\xi_{0}$ then, since $B$ is determined by the function $\nu$ in the sense that $B x=x * \nu, B$ has the same Laplace transform as $\nu$; that is to say, when $\Re z$ is sufficiently large,

$$
\bar{B}(z)=\int_{0}^{\infty} e^{-z t} \nu(t) d t
$$


Therefore, in this case,

$$
\bar{A}(z)=z \bar{B}(z)-\nu(0)=\int_{0}^{\infty} z e^{-z t}\{\nu(t)-\nu(0)\} d t=\int_{0}^{\infty} e^{-z t} d \nu(t),
$$

so that (3.2) holds, the integral being convergent.

We now proceed by induction. Suppose that, for some non-negative integer $n$, (3.2) holds in the sense of summability $(C, n)$ provided that $D^{-n} \nu$ belongs to $\breve{夭}_{0}$ and $\Re z$ is sufficiently large. If $D^{-n-1} \nu$ belongs to $\tilde{F}_{0}$, and $t>0$, then

$$
\begin{aligned}
\int_{0}^{t}\left(1-\frac{u}{t}\right)^{n+1} e^{-z u} d \nu(u)=-\nu(0) & +z \int_{0}^{t}\left(1-\frac{u}{t}\right)^{n+1} e^{-z u} d D^{-1} \nu(u) \\
& +\frac{n+1}{t} \int_{0}^{t}\left(1-\frac{u}{t}\right)^{n} e^{-z u} d D^{-1} \nu(u) .
\end{aligned}
$$

But, by the induction hypothesis (with $D^{-1} \nu$ in place of $\nu$ ),

$$
\bar{B}(z)=\lim _{t \rightarrow \infty} \int_{0}^{t}\left(1-\frac{u}{t}\right)^{n+1} e^{-z u} d D^{-1} \nu(u)=\lim _{t \rightarrow \infty} \int_{0}^{t}\left(1-\frac{u}{t}\right)^{n} e^{-z u} d D^{-1} \nu(u)
$$

when $\mathfrak{R} z$ is sufficiently large; so that

$$
\lim _{t \rightarrow \infty} \int_{0}^{t}\left(1-\frac{u}{t}\right)^{n+1} e^{-z u} d \nu(u)=-\nu(0)+z \bar{B}(z)=\bar{A}(z) .
$$

Thus

$$
\bar{A}(z)=\int_{0}^{\infty} e^{-z t} d \nu(t) \quad(C, n+1),
$$

and the theorem follows.

If $\mathfrak{D}$ is any subspace of $\mathfrak{S}_{0}$ satisfying (1.1) and (1.2), the strongly continuous $V$-operators on $\mathfrak{D}$ form a subalgebra of $\mathfrak{A}(\mathfrak{D})$, say $\mathfrak{R}(\mathfrak{D})$. If $\left(\mathfrak{D}\right.$ is strongly dense in $\mathfrak{F}_{0}$, it follows from Theorem 4 that $\mathfrak{R}(\mathfrak{D})$ effectively consists of those operators in $\mathfrak{N}\left(\mathfrak{S}_{0}\right)$ which leave $\mathfrak{D}$ invariant. In this case, Theorems 8 and 9 show that $\mathfrak{N}(\mathfrak{D})$ is an integral domain (it is commutative, and has no divisors of zero). The full algebra $\mathfrak{N}\left(\mathbb{G}_{0}\right)^{7}$ has the further property that any operator which is inverse to an operator in $\mathfrak{N}\left(\mathfrak{S}_{0}\right)$ is itself in $\mathfrak{N}\left(\mathfrak{C}_{0}\right)$ : this is special case of

THEOREM 12. Let $A$ and $B$ be strongly continuous $V$-operators on a strongly closed subspace $\mathfrak{D}$ of $\mathfrak{F}_{0}$, and suppose that there is an operator $C$ on $\mathfrak{D}$ such that $A=B C$. Suppose also that $B x=0$ only if $x=0$. Then $C$ is a strongly continuous $V$-operator.

$7 \mathfrak{R}\left(\mathfrak{S}_{0}\right)=\mathfrak{M}\left(\mathfrak{S}_{0}\right)$, consisting of the linear combinations of positive $V$-operators on $\mathfrak{夭}_{0}$. 
Proof. If $u>0$ and $x$ is any function in $\mathfrak{D}$ then, since $A$ and $B$ are $V$-operators,

$$
B\left(I_{u} C x-C I_{u} x\right)=I_{u} A x-A I_{u} x=0 ;
$$

so that, by the hypothesis concerning $B, I_{u} C x=C I_{u} x$. In a similar way it can be verified that $C$ is linear, and is therefore a $V$-operator. To show that $C$ is strongly continuous, let $\left\{x_{n}\right\}$ be a strongly convergent sequence in $\mathfrak{D}$ such that the sequence $\left\{C x_{n}\right\}$ is also strongly convergent. Since $A$ and $B$ are strongly continuous,

$$
B\left(\lim _{n \rightarrow \infty} C x_{n}-C \lim _{n \rightarrow \infty} x_{n}\right)=\lim _{n \rightarrow \infty} A x_{n}-A \lim _{n \rightarrow \infty} x_{n}=0,
$$

so that $\lim _{n \rightarrow \infty} C x_{n}=C \lim _{n \rightarrow \infty} x_{n}$; thus the graph of $C$ is closed. Now $\mathfrak{D}$, being strongly closed, is a Fréchet space relative to the strong topology; hence, by Banach's closed-graph theorem $[1,41], C$ is strongly continuous.

4. Operators that commute with convolution. It is a consequence of Theorem 8 that a subspace $\mathfrak{D}$ of $\mathfrak{F}_{0}$, satisfying (1.1) and (1.2), is closed under convolution if it is strongly closed. On the other hand, $\mathfrak{D}_{0}$ is closed under convolution though it is not strongly closed. If $\mathscr{D}$ is any subspace of $\mathfrak{S}_{0}$ which is closed under convolution (so forming an integral domain with no unit element), an operator $A$ on $\mathfrak{D}$ will be said to commute with convolution if

$$
A(x * y)=(A x) * y
$$

for all $x$ and $y$ in D. Such operators are necessarily linear (cf. [5], §4), and, for a given choice of $\mathfrak{D}$, they form an integral domain $\mathfrak{D}^{\sharp}$ in which $\mathfrak{D}$ is isomorphically embedded (by the correspondence $x \rightarrow x *$ ).

A shift operator belongs to $\mathfrak{D}^{\sharp}$ if it maps $\mathfrak{D}$ into itself. Hence if (D) satisfies (1.1) and (1.2), in addition to being closed under convolution, then all the operators in $\mathfrak{D}^{\#}$ are $V$-operators; in fact $\mathfrak{D}^{\sharp}$ is then a maximal commutative subalgebra of $\mathfrak{A}(\mathfrak{D})$. In this case, Theorem 8 shows that every strongly continuous $V$-operator commutes with convolution; so that

$$
\mathfrak{R}(\mathfrak{D}) \subseteq \mathfrak{D} \# \cong \mathfrak{A}(\mathfrak{D}) .
$$

If, further, $\mathfrak{D}$ is strongly closed, then $\mathfrak{N}(\mathfrak{D})=\mathfrak{D} \#$ : for, if $B$ is defined by $B x=x * y$, with $y$ in $\mathfrak{D}$, and $A=B C$, where $C$ is any operator in $\mathfrak{D}^{\sharp}$, then, for any $x$ in $\mathfrak{D}$,

$$
A x=(C x) * y=C(x * y)=C(y * x)=(C y) * x ;
$$

thus the conditions of Theorem 12 are satisfied, so that $C$ belongs to $\mathfrak{N}(\mathfrak{D})$. In particular, the operators on $\mathfrak{F}_{0}$ that commute with convolution 
are precisely the strongly continuous $V$-operators on $\mathfrak{S}_{0}$ (and can therefore be represented according to Theorem 4).

An operator $A$ on $\mathfrak{F}_{0}$ which commutes with convolution can be extended to the whole of $\mathfrak{F}_{0}$ so as to preserve this property. For, if $x$ is any function in $\mathfrak{E}_{0}$, let $x_{n}$ be defined, for each positive integer $n$, as in the proof of Theorem 6: then $x_{n}$ belongs to $\mathfrak{F}_{0}$, and Theorem 1 shows that $A x_{n}(t)$ is independent of $n$ provided that $n \geqq t$; therefore, if $t \geqq 0$, we can define $A x(t)$ to be $A x_{n}(t)$, where $n \geqq t$, without ambiguity. Since convolution is defined locally this extension of $A$ is an operator on $\mathfrak{S}_{0}$ which commutes with convolution. It follows that $A$ is strongly continuous, and that its extension to $\mathfrak{S}_{0}$ is unique (since $\mathfrak{F}_{0}$ is strongly dense in $\left(\mathbb{S}_{0}\right)$.

The integration operator, $D^{-1}$, is an example of an operator on $\sqrt{v}_{0}$ which commutes with convolution. Since $\mathfrak{D}_{0}$ can be expressed as $\bigcap_{n=1}^{\infty} D^{-n} \mathfrak{E}_{0}$, any operator on $\mathfrak{S}_{0}$ which commutes with convolution and leaves $\mathfrak{F}_{0}$ invariant must leave $\mathfrak{D}_{0}$ invariant. The converse of this is false: for, if $A$ is defined by (3.1), $\nu$ being such that $D^{-2} \nu$ belongs to $\mathfrak{F}_{0}$ but $D^{-1} \nu$ does not, and $\nu(0)=0$, then $A$ maps $\mathfrak{D}_{0}$ into itself, by Theorem 10 ; however, if $x(t)=t$ then

$$
A x(t)=\int_{0}^{t}(t-u) d \nu(u)=D^{-1} \nu(t),
$$

so that $x$ is in $\mathfrak{F}_{0}$ but $A x$ is not.

The operators on $\mathfrak{D}_{0}$ that commute with convolution are the perfect operators. These can be characterized as those $V$-operators on $\mathfrak{D}_{0}$ which are continuous in a sense defined in terms of Laplace transforms [7] $]^{8}$. The strongly continuous perfect operators are the strongly continuous $V$-operators on $\mathfrak{D}_{0}$, constituting the algebra $\mathfrak{N}\left(\mathfrak{D}_{0}\right)$; this algebra, and also its subalgebra $\mathfrak{M}\left(\mathfrak{D}_{0}\right)$, can be characterized in terms of convolution, as follows.

THEOREM 13. A perfect operator belongs to $\mathfrak{N}\left(\mathfrak{D}_{0}\right)$ if and only if it can be extended to the whole of $\mathfrak{F}_{0}$ so as to commute with convolution; it belongs to $\mathfrak{M}\left(\mathfrak{D}_{0}\right)$ if and only if this extension (necessary unique) leaves $\mathfrak{F}_{0}$ invariant.

Proof. If an operator $A$ on $\mathfrak{D}_{0}$ can be extended to $\mathfrak{S}_{0}$ so as to commute with convolution, then its extension belongs to $\mathfrak{N}\left(\mathfrak{S}_{0}\right)$, so that $A$ itself belongs to $\mathfrak{N}\left(\mathfrak{D}_{0}\right)$. On the other hand, any operator $A$ in $\mathfrak{N}\left(\mathfrak{D}_{0}\right)$ admits the representation (3.1), which provides an extension of $A$ to $\mathbb{S}_{0}$ : this extension, being strongly continuous, commutes with convolution;

8 It is not at present known whether there are any $V$-operators on $\mathscr{D}_{0}$ which are not perfect; that is to say, it is not known whether $\mathfrak{U}\left(\mathscr{D}_{0}\right)$ is commutative or not (but there are linear operators on $\mathfrak{D}_{0}$ which commute with $D$ and are not perfect $[\mathbf{6}]$ ). 
it is also unique, since $\mathfrak{D}_{0}$ is strongly dense in $\mathbb{F}_{0}$.

If a perfect operator $A$ has a strongly continuous extension to $\mathbb{F}_{0}$ which leaves $\mathfrak{F}_{0}$ invariant, we can regard $A$ as a $V$-operator on $\mathfrak{F}_{0}$; then, by Theorem 3, there is a real number $c$ such that $\|A\|_{t}=O\left(e^{c t}\right)$ as $t \rightarrow \infty$, and this implies, by Theorem 7, that $A$ belongs to $\mathfrak{M}\left(\mathfrak{D}_{0}\right)$. On the other hand, if $A$ belongs to $\mathfrak{M}\left(\mathfrak{D}_{0}\right)$ then the extension of $A$ to $\mathbb{F}_{0}$ given by (3.1) leaves $\xi_{0}$ invariant, by Theorem 3 of [5].

Finally, we give an example of a $V$-operator, on a strongly dense subspace of $\mathfrak{S}_{0}$, which does not commute with convolution. Let $h$ be the Heaviside unit function $(h(t)=1$ if $t \geqq 0)$, and let $\mathscr{D}_{1}$ be the class of all functions $x$ given by

$$
x=D^{-1}(y+B h),
$$

where $y$ belongs to $\mathfrak{C}_{0}$ and $B$ is an operator of the type (2.1). Then $\mathfrak{D}_{0} \subseteq \mathfrak{D}_{1} \subseteq \mathfrak{C}_{0}$, and $\mathfrak{D}_{1}$ satiyfies (1.1) and (1.2); moreover, $\mathfrak{D}_{1}$ is closed under convolution. It is clear that $y$ and $B$ in (4.1) are uniquely determined by $x$, and that the mapping $x \rightarrow y$ is a $V$-operator, say $A$, on $\mathfrak{D}_{1}$. The operator $D^{-1}$ maps $\mathfrak{D}_{1}$ into itself and commutes with convolution. However, $A D^{-1} x=x$ and $D^{-1} A x=y$, so that $A D^{-1} \neq D^{-1} A$. Hence $A$ does not commute with convolution. It follows that the algebra $\mathfrak{A}\left(\mathscr{D}_{1}\right)$, of all $V$-operators on $\mathfrak{D}_{1}$, is not commutative.

\section{REFERENCES}

1. S. Banach, Théorie des opérations linéaires, Warsaw, 1932.

2. R. E. Edwards, Representation theorems for certain functional operators, Pacific J. Math., 7 (1957), 1333-1339.

3. H König und J. Meixner, Lineare Systeme und lineare Transformationen, Math. Nachrichten, 19 (1958), 265-322.

4. E. C. Titchmarsh, Theory of Fourier integrals, Oxford, 1937.

5. J. D. Weston, Positive perfect operators, Proc. London. Math. Soc. (3), 10 (1960), 545-565.

6. Characterizations of Laplace transforms and perfect operators, Archive for Rational Mechanics and Analysis, 3 (1959), 348-354.

7. - Operational calculus and generalized functions, Proc. Roy. Soc. A, 250 (1959), 460-471.

8. A. C. Zaanen, Theory of integration, Amsterdam, 1958.

UNIVERSity OF DURHAM, NeWCASTLE UPON TYNE

AND

California Institute of Technology, Pasadena 


\section{PACIFIC JOURNAL OF MATHEMATICS}

\section{EDITORS}

David GILbarg

Stanford University

Stanford, California

\section{F. H. BRowneLL}

University of Washington

Seattle 5, Washington

\section{A. L. Whiteman}

University of Southern California Los Angeles 7, California

\section{J. PAIGe}

University of California

Los Angeles 24, California

\section{ASSOCIATE EDITORS}
E. F. BECKENBACH
T. M. CHERRY
D. DERRY

\author{
E. HEWITT \\ A. HORN \\ L. NACHBIN
}

\author{
M. OHTSUKA \\ H. L. ROYDEN \\ M. M. SCHIFFER
}

E. SPANIER

E. G. STRAUS

F. WOLF

\section{SUPPORTING INSTITUTIONS}

\author{
UNIVERSITY OF BRITISH COLUMBIA \\ CALIFORNIA INSTITUTE OF TECHNOLOGY \\ UNIVERSITY OF CALIFORNIA \\ MONTANA STATE UNIVERSITY \\ UNIVERSITY OF NEVADA \\ NEW MEXICO STATE UNIVERSITY \\ OREGON STATE COLLEGE \\ UNIVERSITY OF OREGON \\ OSAKA UNIVERSITY \\ UNIVERSITY OF SOUTHERN CALIFORNIA
}

\author{
STANFORD UNIVERSITY \\ UNIVERSITY OF TOKYO \\ UNIVERSITY OF UTAH \\ WASHINGTON STATE COLLEGE \\ UNIVERSITY OF WASHINGTON \\ AMERICAN MATHEMATICAL SOCIETY \\ CALIFORNIA RESEARCH CORPORATION \\ HUGHES AIRCRAFT COMPANY \\ SPACE TECHNOLOGY LABORATORIES \\ NAVAL ORDNANCE TEST STATION
}

\footnotetext{
Mathematical papers intended for publication in the Pacific Journal of Mathematics should be typewritten (double spaced), and the author should keep a complete copy. Manuscripts may be sent to any one of the four editors. All other communications to the editors should be addressed to the managing editor, L. J. Paige at the University of California, Los Angeles 24, California.

50 reprints per author of each article are furnished free of charge; additional copies may be obtained at cost in multiples of 50 .
}

The Pacific Journal of Mathematics is published quarterly, in March, June, September, and December. The price per volume (4 numbers) is $\$ 12.00$; single issues, $\$ 3.50$. Back numbers are available. Special price to individual faculty members of supporting institutions and to individual members of the American Mathematical Society: $\$ 4.00$ per volume; single issues, $\$ 1.25$.

Subscriptions, orders for back numbers, and changes of address should be sent to Pacific Journal of Mathematics, 2120 Oxford Street, Berkeley 4, California.

Printed at Kokusai Bunken Insatsusha (International Academic Printing Co., Ltd.), No. 6, 2-chome, Fujimi-cho, Chiyoda-ku, Tokyo, Japan.

PUBLISHED BY PACIFIC JOURNAL OF MATHEMATICS, A NON-PROFIT CORPORATION

The Supporting Institutions listed above contribute to the cost of publication of this Journal, but they are not owners or publishers and have no responsibility for its content or policies. 


\section{Pacific Journal of Mathematics}

\section{Vol. 10, No. $4 \quad$ December, 1960}

M. Altman, An optimum cubically convergent iterative method of inverting a linear bounded operator in Hilbert space . . . . . . . . . . . . . . . . . . . . . . . . . . 1107

Nesmith Cornett Ankeny, Criterion for rth power residuacity ................. 1115

Julius Rubin Blum and David Lee Hanson, On invariant probability measures I . . . . . 1125

Frank Featherstone Bonsall, Positive operators compact in an auxiliary topology ..... 1131

Billy Joe Boyer, Summability of derived conjugate series . . . . . . . . . . . . . . . . 1139

Delmar L. Boyer, A note on a problem of Fuchs . . . . . . . . . . . . . . . . . 1147

Hans-Joachim Bremermann, The envelopes of holomorphy of tube domains in infinite

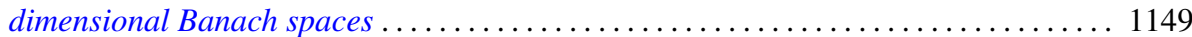

Andrew Michael Bruckner, Minimal superadditive extensions of superadditive

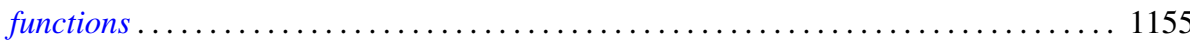

Billy Finney Bryant, On expansive homeomorphisms .................... 1163

Jean W. Butler, On complete and independent sets of operations in finite algebras . . . . . 1169

Lucien Le Cam, An approximation theorem for the Poisson binomial distribution ...... 1181

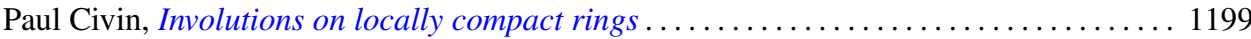

Earl A. Coddington, Normal extensions of formally normal operators . . . . . . . . . 1203

Jacob Feldman, Some classes of equivalent Gaussian processes on an interval ........ 1211

Shaul Foguel, Weak and strong convergence for Markov processes . . . . . . . . . . . 1221

Martin Fox, Some zero sum two-person games with moves in the unit interval ........ 1235

Robert Pertsch Gilbert, Singularities of three-dimensional harmonic functions . . . . . . . 1243

Branko Grünbaum, Partitions of mass-distributions and of convex bodies by

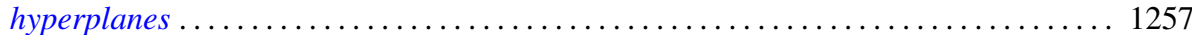

Sidney Morris Harmon, Regular covering surfaces of Riemann surfaces ........... 1263

Edwin Hewitt and Herbert S. Zuckerman, The multiplicative semigroup of integers

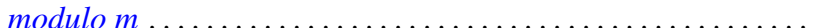

Paul Daniel Hill, Relation of a direct limit group to associated vector groups . ......... 1309

Calvin Virgil Holmes, Commutator groups of monomial groups . .

James Fredrik Jakobsen and W. R. Utz, The non-existence of expansive homeomorphisms

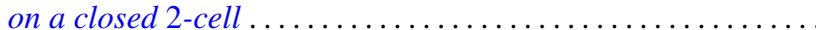

John William Jewett, Multiplication on classes of pseudo-analytic functions . . . . . . . 1323

Helmut Klingen, Analytic automorphisms of bounded symmetric complex domains . . . . 1327

Robert Jacob Koch, Ordered semigroups in partially ordered semigroups . . . . . . . . 1333

Marvin David Marcus and N. A. Khan, On a commutator result of Taussky and

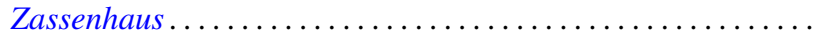

John Glen Marica and Steve Jerome Bryant, Unary algebras......

Edward Peter Merkes and W. T. Scott, On univalence of a continued fraction . . . . . . . 1361

Shu-Teh Chen Moy, Asymptotic properties of derivatives of stationary measures . . . . . 1371

John William Neuberger, Concerning boundary value problems . . . . . . . . . . . 1385

Edward C. Posner, Integral closure of differential rings . . . . . . . . . . . . . . . . . 1393

Marian Reichaw-Reichbach, Some theorems on mappings onto . . . . . . . . . . . . . 1397

Marvin Rosenblum and Harold Widom, Two extremal problems . . . . . . . . . . . . . . . . 1409

Morton Lincoln Slater and Herbert S. Wilf, A class of linear differential-difference

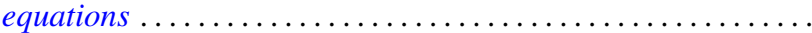

Charles Robson Storey, Jr., The structure of threads . . . . . . . . . . . . . . . . . . 1429

J. François Treves, An estimate for differential polynomials in $\partial / \partial z_{1},, \cdots, \partial / \partial z_{-} n \ldots \ldots 1447$

J. D. Weston, On the representation of operators by convolutions integrals . . . . . . . . 1453

James Victor Whittaker, Normal subgroups of some homeomorphism groups ......... 1469 